\title{
On the Number of Directions in Visibility Representations of Graphs * (Extended Abstract)
}

\author{
Evangelos Kranakis ${ }^{1}$ (kranakis@scs.carleton.ca) \\ Danny Krizanc ${ }^{1}$ (krizanc@scs.carleton.ca) \\ Jorge Urrutia ${ }^{2}$ (jorge@csi.uottawa.ca) \\ 1 Carleton University, School of Computer Science, Ottawa, ON, Canada \\ 2 University of Ottawa, Department of Computer Science, Ottawa, ON, Canada
}

\begin{abstract}
We consider visibility representations of graphs in which the vertices are represented by a collection $\mathcal{O}$ of non-overlapping convex regions on the plane. Two points $x$ and $y$ are visible if the straight-line segment $x y$ is not obstructed by any object. Two objects $A, B \in \mathcal{O}$ are called visible if there exist points $x \in A, y \in B$ such that $x$ is visible from $y$. We consider visibility only for a finite set of directions. In such a representation, the given graph is decomposed into a union of unidirectional visibility graphs, for the chosen set of directions. This raises the problem of studying the number of directions needed to represent a given graph. We study this number of directions as a graph parameter and obtain sharp upper and lower bounds for the representability of arbitrary graphs.

1980 Mathematics Subject Classification: 68R10, 68U05

CR Categories: F.2.2

Key Words and Phrases: Graph, Number of directions, Polygon, Visibility.
\end{abstract}

\section{Introduction}

We consider visibility representations of graphs in which the vertices are represented by a collection $\mathcal{O}$ of non-overlapping convex regions on the plane. Two points $x$ and $y$ are visible if the straight-line segment $x y$ is not obstructed by any object $A \in \mathcal{O}$. Two objects $A, B \in \mathcal{O}$ are called visible if there exist points $x \in A, y \in B$ which are visible. This gives rise to a graph with vertices the given objects. For a given direction, two such objects are "adjacent" if and only if one object is visible from the other along the given direction. It is well-known and also easy to prove that graphs which admit such a unidirectional representation must be planar $[6,8]$. There have been several results in the literature which investigate the recognition problem for such a graph representation. Complete

\footnotetext{
^ Research supported in part by NSERC (Natural Sciences and Engineering Research Council of Canada) grant.
} 
characterizations exist for unidirectional representations as well as partial results for 2-directional representations $[1,5,6,8]$.

Every graph can be represented as the visibility graph of a collection of nonoverlapping convex regions in such a way that the given graph is decomposed into a union of unidirectional visibility graphs for an appropriately chosen set of directions. Indeed, draw the graph on the plane such that the edges are straight line segments and no three vertices are on the same line. Now replace the vertices with sufficiently small circles. Clearly, every graph can be represented with $|E|$ directions, where $E$ is the set of edges.

This raises the problem of studying the number of directions needed to represent a given graph. We study this number of directions as a graph parameter and obtain upper and lower bounds for arbitrary as well as specific graphs. We call a graph $k$-directional if it can be represented this way with at most $k$ directions.

The usual criteria for drawing a graph involve minimizing parameters such as number of directions of edges, as well as "misalignments". In particular, this makes desirable visibility representations with "low" number of directions. Our result implies that there exist $n$ vertex graphs for which "low" $\geq n^{2} / \log n$.

\subsection{Definitions}

Let $\mathcal{O}$ be a collection of $n$ non-overlapping, convex regions on the plane. For any given direction $\phi$ we define the visibility graph among these objects along direction $\phi$ as follows:

1. vertices of the graph are the $n$ objects,

2. for $a, b$ objects, $\{a, b\}$ is an edge if and only if $a$ is visible from $b$ along direction $\phi$.

As pointed out before, for every graph $G=(V, E)$ we can find a collection of $n$ plane, non-overlapping convex objects such that $G$ can be represented as the union of graphs $G_{\phi}=\left(V, E_{\phi}\right), \phi \in \Phi$, where $\Phi$ is an appropriately selected set of directions. We denote with $\operatorname{dir}(G)$ the minimum number $|\Phi|$ of directions needed to represent $G$. It is clear from the definitions that $\operatorname{dir}(G) \leq|E|$.

In addition, we will also consider the case where only directions are considered for which no two objects have the same supporting line. We denote with $\operatorname{dir}^{*}(G)$ the minimum number $|\Phi|$ of directions needed to represent $G$ in this way. A related graph parameter is the thicknes of a graph $G$, denoted by $\theta(G)$, and is defined in [3] as follows: it is the smallest number of planar subgraphs whose union is $G$. A well-known result $[6,8]$ states that unidirected graphs are planar. This implies that $\theta(G) \leq \operatorname{dir}(G) \leq \operatorname{dir}^{*}(G) \leq|E|$.

In general, we make the assumption that our objects are compact convex sets. For a given direction and any convex object on the plane it is clear that the visibility of the object is fully determined by the line segment which is delimited by the convex object and the tangents to the object parallel to the given direction (see Figure 1). For this reason (as it is customary) for any fixed direction we will represent the objects as line segments perpendicular to the given direction. 


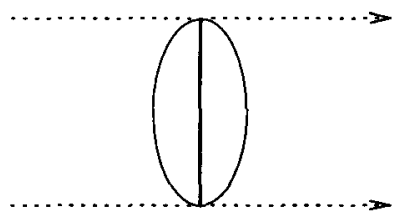

Fig. 1. Reducing objects to straight-line segments.

In a similar manner we can define the quantities $\operatorname{dir}_{\text {seg }}(G), \operatorname{dir}_{\text {seg }}^{*}(G)$, where the set $\mathcal{O}$ of objects consists of finite straight-line segments. Although not explicitely proved, the results of Section 2 are still valid for these parameters. The necessary modifications are left to the reader.

\subsection{Results of the paper}

Here is a summary of the results of the paper. In Section 2 we consider techniques for determining the size of $\operatorname{dir}(G)$ for arbitrary graphs $G$. The quantity $\operatorname{dir}^{*}(G)$ has a different behavior. It turns out that one can obtain sharper bounds on $\operatorname{dir}^{*}(G)$ when the given graph has girth at least four. This enables us to determine the number of directions for several special graphs, like multidimensional grids, complete bipartite, etc. In Section 3 we give strong lower bounds on the number of directions needed to represent a graph when the objects are polygons. In particular, we show the existence of $n$ vertex graphs $G$ such that $\operatorname{dir}_{s e g}(G)=$ $\Omega\left(n^{2} / \log n\right)$. In Section 4 we consider unidirectional representations of graphs in three dimensions where the objects are polygons parallel to the $x y$-plane. We show that all hamiltonian graphs have such a representation and give a tight bound on the number of vertices of the polygons.

\section{Number of Directions}

In this section we obtain estimates on the parameters $\operatorname{dir}(G)$ and $\operatorname{dir}^{*}(G)$. For any graph $G$ let $\bar{G}$ denote the complement of $G$; this has the same set of vertices, but two points are adjacet in $\bar{G}$ if and only if they are not adjacent in $G$.

Theorem 1. For any graph $G$, either $\operatorname{dir}(G)=\Omega(n)$ or $\operatorname{dir}(\bar{G})=\Omega(n)$.

Proof (Outline) The theorem will follow from the following lemmas.

Lemma 2 [6, 8]. Unidirectional graphs are planar.

Lemma 3. For any graph $G=(V, E)$ on $n$ vertıces we have that $\operatorname{dir}(G) \geq$ $|E| / 3(n-2)$. 
Proof Let $\Phi$ be a set of directions representing the graph $G$ for a given set of objects. Let $E_{\phi}$ be the set of edges corresponding to direction $\phi$. We know that $E=\bigcup_{\phi \in \Phi} E_{\phi}$ and therefore $|E|=\sum_{\phi \in \Phi}\left|E_{\phi}\right|$. However each graph $G_{\phi}$ is planar. Hence $\left|E_{\phi}\right| \leq 3(n-2)$, which proves the desired result.

Now we can finish the proof of Theorem 1 . Let $G=(V, E)$ be a given graph. If $|E| \geq n(n-1) / 4$ then by Lemma $3 \operatorname{dir}(G)=\Omega(n)$. Otherwise, the complement of $G$ must have at least $n(n-1) / 4$ edges, in which case the same lemma applies. This proves the theorem.

For graphs with girth $\geq 4$ we can prove a better lower bound.

Theorem 4. For any graph $G=(V, E)$ on $n$ vertices and girth at least 4 we have that $\operatorname{dir}^{*}(G) \geq|E| /(n-1)$.

Proof (OUturne) First we need the following simple lemma.

Lemma $5[6,8]$. No cycle of more than 3 nodes is representable with only one direction, when no two segment endpoints form a line parallel to the given direction.

To prove the main theorem we argue as before. Let $\Phi$ be a set of directions representing the graph $G$ for a given set of objects. Let $E_{\phi}$ be the set of edges corresponding to direction $\phi$. We know that $E=\bigcup_{\phi \in \Phi} E_{\phi}$ and therefore $|E|=$ $\sum_{\phi \in \Phi}\left|E_{\phi}\right|$. Since the girth of $G$ is $\geq 4$ the graph $G_{\phi}$ (see Lemma 5) cannot have any cycles. Hence it is a tree and we have that $\left|E_{\phi}\right| \leq n-1$. This proves the theorem.

Note that the quantities $\operatorname{dir}(G)$ and $\operatorname{dir}^{*}(G)$ may be unequal. This is due to the fact that although all cycles are unidirectional (see Figure 2), every cycle $C$

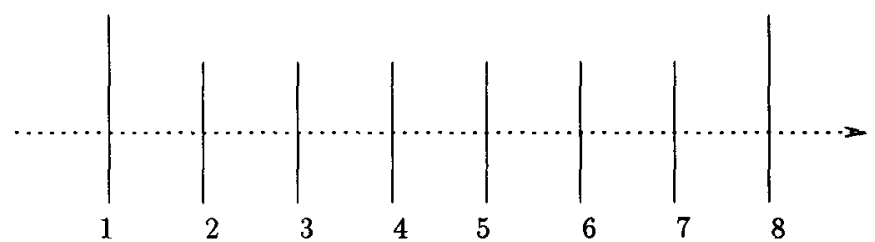

Fig. 2. Representing the 8-node cycle with one direction.

of more than three nodes must satisfy $\operatorname{dir}^{*}(C)>1$.

The parameter $\operatorname{dir}^{*}(G)$ is not monotonic for the subgraph property, i.e. if $G$ is a subgraph of $G^{\prime}$ then it is not in general true that $\operatorname{dir}^{*}(G) \leq \operatorname{dir}^{*}\left(G^{\prime}\right)$. To see this take an arbitrary planar graph $G$ with girth 4 . Such a graph requires at least 2 directions. For any interior face add a vertex and connect this vertex with every vertex of the face. The resulting graph $G^{\prime}$ is triangulated and planar, hence $\operatorname{dir}^{*}(G)=1$. However, it is easy to see that for any graph $G$, if the clique $K_{r}$ is a subgraph of $G$ then $\operatorname{dir}^{*}\left(K_{r}\right) \leq \operatorname{dir}^{*}(G)$. 


\subsection{Special graphs}

An interesting case occurs when we consider the number of directions for representing the product of graphs (for the definitions see [3]). We say that the representation $\mathcal{O}$ of a graph has a visibility gap if there are two directions $\phi_{1}, \phi_{2}$ such that for every $x \in O \in \mathcal{O}$ any line through $x$ with direction $\phi, \phi_{1} \leq \phi \leq \phi_{2}$, intersects no object of $\mathcal{O}$ except $O$. Now we can prove the following lemma.

Lemma 6. Let $P_{n}$ denote the path on $n$ vertices. For any graph $G$ which has a visibility gap,

$$
\operatorname{dir}^{*}\left(P_{n} \times G\right) \leq 1+\operatorname{dir}^{*}(G) .
$$

Proof (Outline) Consider a representation of the graph $G$ with a set of direc-

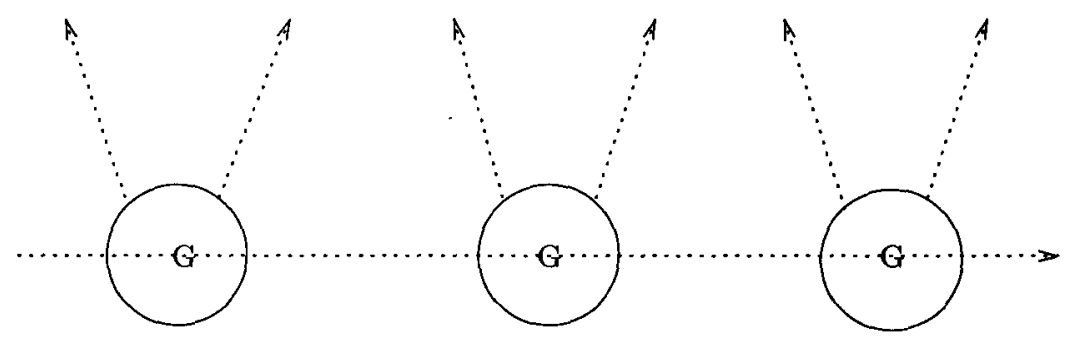

Fig. 3. Representing the cartesian product graph $L_{n} \times G$.

tions $\Phi$ such that $\operatorname{dir}(G)=|\Phi|$ for a given collection of objects. By applying an appropriate linear geometric transformation we can reduce as much as we wish the span of the directions of the graph, i.e. we can assume that $\Phi \subseteq(\pi / 2-\epsilon, \pi / 2+\epsilon)$, where $\epsilon>0$ is sufficiently close to $\pi / 2$. We arrange $n$ copies of the graph $G$ on the path $P_{n}$ such that in no two different graphs are the corresponding copies of the objects pairwise visible along the directions in $\Phi$. Using the fact that the graph $G$ has a visibility gap, we can add a new direction $\phi=0$ and guarantee that the copies of the convex objects are such that the corresponding weak visibility graph is the cartesian product $P_{n} \times G$ (see Figure 3 ).

For special graphs like multidimensional grids, hypercubes, etc we can give more precise bounds on the number of directions needed for their visibility representation. Namely, we can prove the following result.

\section{Theorem 7 .}

1. $\lfloor(n+7) / 6\rfloor \leq \operatorname{dir}\left(K_{n}\right) \leq\lfloor n / 2\rfloor$.

2. $\frac{m n}{m+n-1} \leq \operatorname{dir}^{*}\left(K_{m, n}\right) \leq \min \{m, n\}$.

3. $\operatorname{dir}^{*}\left(M_{n}^{d}\right)=d$, for $n$ sufficiently large, where $M_{n}^{d}$ is the d dimensional grid of side $n$. 
4. $\lceil(n+1) / 4\rceil \leq \operatorname{dir}\left(Q_{n}\right) \leq\lceil n / 3\rceil$ and $\lceil n / 2\rceil \leq \operatorname{dir}^{*}\left(Q_{n}\right)$, where $Q_{n}$ is the $n$-dimensional hypercube.

Proof (Outline) We give only an outline of the required constructions. An $n(n-1) / 6(n-2)$ lower bound in part (1) follows directly from Lemma 3. Using the formula for the thickness of $K_{n}$ (see [3][page 120]) we can improve this to $\lfloor(n+7) / 6\rfloor$. The upper bound is proved by induction on $n$ (see Figure 4). We

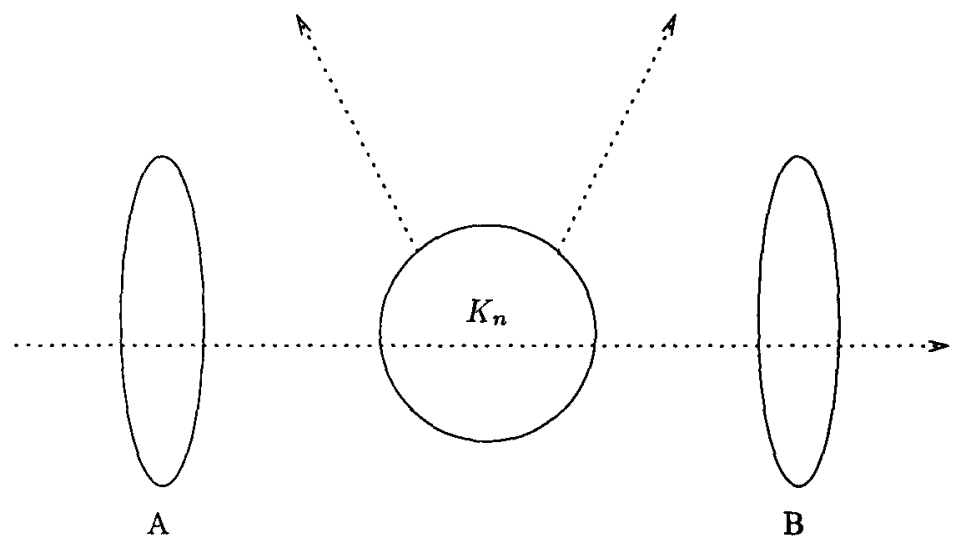

Fig. 4. Representing the complete graph $K_{n+2}$.

show how to construct a representation of $K_{n+2}$ from a representation of $K_{n}$ by adding only one new direction. As in Lemma 6 we may apply a geometric transformation that reduces the span of the set of directions of $K_{n}$. Assume that the objects representing $K_{n}$ are sufficiently small and add two new objects $A, B$ as depicted in Figure 4 . The result now follows by induction and from the fact that $\operatorname{dir}\left(K_{2}\right)=\operatorname{dir}\left(K_{3}\right)=1$.

The lower bounds in parts (2) and (3) follow directly from Theorem 4, The upper bound of $\operatorname{dir}^{*}\left(M_{n}^{d}\right)$ follows from the fact that the $d$-dimensional grid $M_{n}^{d}$ is the direct product graph of $d$ copies of $P_{n}$ and Lemma 6. The upper bound for part (2) follows from the representation depicted in Figure 5. The upper bound $\operatorname{dir}\left(Q_{n}\right) \leq\lceil n / 3\rceil$ follows from the fact that $\operatorname{dir}\left(Q_{3}\right)=1$ (since $Q_{3}$ is a hamiltonian graph) and using the analogue of Lemma 6 for the product graph $Q_{3} \times G$ (i.e. $\operatorname{dir}\left(Q_{3} \times G\right) \leq 1+\operatorname{dir}(G)$, provided that $G$ has a visibility gap). The lower bound for $\operatorname{dir}\left(Q_{n}\right)$ follows from the fact that $\theta\left(Q_{n}\right)=\lceil(n+1) / 4\rceil$ (see [3][page 121]). 


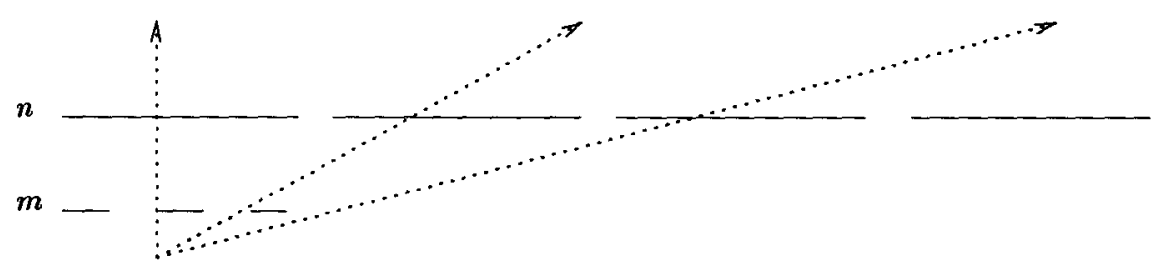

Fig. 5. Representing the complete bipartite graph $K_{m, n}$.

\section{Lower Bounds}

In this section we use a result of $\mathrm{H}$. Warren [10] on the number of sign patterns of a set of polynomials in order to prove the existence of graphs whose representation requires superlinear number of directions.

Let $p_{1}, p_{2}, \ldots, p_{m}$ be polynomials in $r$ variables and for $x=\left(x_{1}, \ldots, x_{r}\right)$ let the sign-pattern at $x$ be the vector $\left(\operatorname{sgn} p_{1}(x), \ldots, \operatorname{sgn} p_{m}(x)\right)$ consisting of +1 and -1 . Let $s\left(p_{1}, \ldots, p_{m}\right)$ be the number of different sign-patterns for all values of $\boldsymbol{x} \in \mathbf{R}^{r}$.

Our main theorem makes use of the following result of H. Warren [10].

Lemma 8. If $p_{1}, \ldots, p_{m}$ are polynomials in $r$ variables with degree $\leq d$ then the number of sign-patterns is $s\left(p_{1}, \ldots, p_{m}\right) \leq\left(\frac{4 \mathrm{edm}}{r}\right)^{r}$.

The following theorem is the main result of this section.

Theorem 9. There exist n-vertex graphs $G \in \mathcal{G}$ such that

$$
\operatorname{dir}_{s e g}(G)=\Omega\left(n^{2} / \log n\right) .
$$

Proof (OutLine) Suppose that $k$ directions are sufficient to represent every graph. We consider the case where the objects representing the graphs are line segments. There are $n$ finite, straight line segments determined by their endpoints. For any given direction the visibility of any pair of line segments is determined by whether or not a straight line with slope the given direction and emanating from one straight line segment intersects the other straight line segment. This easily gives rise to $O\left(n^{2} k\right)$ polynomial conditions of constant degree in at most $4 n+2 k$ variables. By Warren's theorem the number of sign patterns is bounded above by

$$
\left(\frac{n^{2} k C}{4 n+2 k}\right)^{4 n+2 k}
$$

where $C$ is a constant. In order to represent all possible graphs this quantity must be at least $2\left(\begin{array}{l}n \\ 2\end{array}\right)$. Taking logarithms and ignoring lower order terms this means that $k=\Omega\left(n^{2} / \log n\right)$. This proves the theorem. 
A similar theorem holds when the objects are (smooth) convex sets determined by a constant number of variables (e.g. circles, ellipses, etc). A similar lower bound is also possible if the objects representing the graphs are convex polygons. More precisely, as in Theorem 9, we can prove the following result.

Theorem 10. There exist n-vertex graphs $G$ requiring $\Omega\left(\frac{n^{2}}{\log n}-m\right)$ directions for their visibility representation by convex polygons whose total number of edges is $m \geq n$.

\section{Three dimensional representations}

In contrast to the two dimensional case, we can obtain unidirectional representations in three dimensional space for a large class of graphs. Here we assume that the objects are polygons which are parallel to the $x y$-plane. In the proofs below it will be convenient to use the standard terminology and definitions on the page number of a graph $[4,12]$.

Theorem 11. Every graph $G$ which has a hamiltonian path has a unidirectional representation in three dimensional space, where each object is a convex polygon with at most $\lfloor n / 2\rfloor$ vertices, and this bound is tight.

Proof (Outline) We give only an outline of the algorithm. First we show how to represent hamiltonian, one-page graphs. Initially, we represent the vertices of the graph as segments of equal length. The segments are parallel and their corresponding endpoints form two parallel straight lines. This represents the hamiltonian path. Next, beginning with the outermost edges of the graph we augment appropriately the lengths of the adjacent vertices so that the visibility representation of the segments coincides with the representation of the given graph. Clearly, this shows that hamiltonian, one-page graphs have a unidirectional representation with parallel segments.

We use the transformation above for graphs of arbitrary page number $p$. We represent each page with parallel segments and join the corresponding vertices to form polygons with $p$ vertices. In general, these will be star-polygons. However, it is easy to see that in the transformation of the previous paragraph we can adjust at will the range of length of the segments representing our graph. This easily implies that the corresponding polygons may be assumed to be convex with $p$ vertices (notice that if $p=2$ then the representation can be assumed to be planar).

First we prove an $n-1$ upper bound. It is easy to see that the complete graph $K_{n}$ can be represented with $n-1$ pages, where the vertices on the spine form a hamiltonian path. To represent $G$ put a hamiltonian path of $G$ on the spine and delete edges of $K_{n}$ which are not edges of $G$. This gives a representation of $G$ on at most $n-1$ pages. Now the previous argument implies the desired result.

The proof of the $\lfloor n / 2\rfloor$ upper bound is based on a partition of the set of edges of the complete graph into $\lfloor n / 2\rfloor$ paths. The partition can be such that 
each path lies on a page. This and the previous argument implies the required bound for any graph $G$. The reason this bound is tight is that $K_{n}$ has exactly $n(n-1) / 2$ edges and, not counting the edges on the spine, no page can have more than $n-1$ edges.

We can give a related theorem for the case of planar graphs, where the visibility representation uses star polygons having $O(1)$ vertices. Namely we can prove the following result.

Theorem 12. Every planar graph $G$ has a unidirectional representation in three dimensional space, where each object is a 10-vertex star polygon.

Proof (Outline) We give an outline of the algorithm. The representation with star-shaped polygons follows by induction on the number of vertices of the planar graph. First we give a representation with overlapping star-polygons on the plane; here adjacency means that two polygons overlap. By "lifting" the polygons in parallel in three dimensional space we get the desired representation.

We use the fact that every planar graph has a vertex of degree at most 5. Delete such a vertex from the given planar graph. The remaining graph has a representation satisfying the conditions of the theorem. Now add the deleted vertex and represent it as a star polygon which overlaps only its adjacent star polygons. This gives the desired representation.

An even stronger theorem has been independently proved in [2], namely every planar graph has a uni-directional representation in three dimensional space by rectangles parallel to the $x y$-plane, where the visibility direction is parallel to the $z$-axis.

\section{Conclusion}

In this paper we have studied the number of directions needed in the visibility representations of graphs. We have obtained several sharp upper and lower bounds for general as well as special graphs. Several interesting questions concern tightening the existing upper and lower bounds. For example, as shown in Section 3, Theorem 8 implies there exist graphs with lower bound $n^{2} / \log n$ on the quantity dir seg. We conjecture that this lower bound is tight. A similar question applies when the objects are (smooth) convex sets: Is it true that $\operatorname{dir}(G) \leq n$, for every graph $G$ ? It is known that every planar graph is a subgraph of a unidirectional visibility graph on the plane by using line segments [8]. However we conjecture that there is a constant $k$ such that every planar graph is a $k$-directional visibility graph (here visibility refers to the notion of visibility as defined in this paper).

Another interesting problem concerns the complexity of the problem $\operatorname{dir}(G) \leq$ $k$, for $G$ a graph on $n$ nodes and $k$ constant (or variable). In particular, is there an efficient (even approximation) algorithm for computing the function $\operatorname{dir}(G)$ ? We conjecture that this is not true.

A rather intriguing question concerns the study of the number of directions needed for the visibility representation of the product graph $H \times G$ in terms 
of $\operatorname{dir}(H), \operatorname{dir}(G)$. For example, is it true that $\operatorname{dir}(H \times G) \leq \operatorname{dir}(H)+\operatorname{dir}(G)$ ? (Similar question for $\operatorname{dir}^{*}$.)

\section{Acknowledgements}

Many thanks to Mark Noy for suggesting the use of Lemma 8, Michel Pocchiola for useful conversations, and Roberto Tamassia for pointing out relevant references.

\section{References}

1. G. Di Battista and P. Eades and R. Tamassia and I. G. Tollis, "Algorithms for Drawing Graphs: An Annotated Bibliography", Comput. Geom. Theory Appl, to appear. (Preprint available by anonymous ftp from ftp.cs.brown. edu: pub/papers/compgeo/.)

2. P. Bose, H. Everett, S. Fekete, A. Lubiw, H. Meijer, K. Romanik, T. Shermer, and S. Whitesides, "On a Visibility Representation for Graphs in Three Dimensions" (abstract), ALCOM International Workshop on Graph Drawing and Topological Graph Algorithms, Paris, September 26-29, 1993, pp. 53-54. (Also, in McGill Technical Report "Snapshots of Computational and Discrete Geometry", 1994.)

3. F. Harary, "Graph Theory", Addison-Wesley Publishing Company, 1969.

4. L. S. Heath and S. Istrail, "The Pagenumber of Genus $g$ Graphs is $O(g)$ ", in STOC 87 , pages $388-397$.

5. J. O'Rourke, "Computational Geometry Column 18", International Journal of Computational Geometry \& Applications, pp. 107-113, Vol. 3, No. 1, 1993.

6. I. Rival and J. Urrutia, "Representing Orders on the Plane by Translating Convex Figures", Order 4 (1988), 319-329.

7. I. Rival and J. Urrutia, "Representing Orders by Moving Figures in Space", Discrete Mathematics 109 (1992), 255-263.

8. R. Tamassia and I. G. Tollis, "A Unified Approach to Visibility Representations of Planar Graphs", Discrete Comput. Geom. 1:321-341, 1986.

9. R. Tamassia and I. G. Tollis, "Plane Representations of Graphs and Visibility Between Parallel Segments", TR ACT-37, Univ. of Ill., Urbanna Champaign, 1985.

10. H. Warren, "Lower Bounds for Approximation by Nonlinear Manifolds", Transactions of the AMS 133(1968), 167-178.

11. S. K. Wismath, "Characterizing Bar Line-of-sight Graphs", Proc. 1st Annu. ACM Sympos. Comput. Geom., pp. 147-152, 1985.

12. M. Yannakakis, "Four Pages are Necessary and Sufficient for Planar Graphs", in STOC86, pages $104-108$. 